\title{
Standard Graphic Symbols
}

\author{
BY EDWARD J. CHENEY \\ Chief, Div. of Light, Heat and Power, Public Service Commission, Albany, N. Y.
}

\begin{abstract}
Symbols have been in vogue since the early ages and are particularly useful to the electrical engineer. Those now in use are the result of growth and evolution and there is no uniformity or agreement. It is desirable to standardize them as far as possible in order to afford simplicity and general usefulness, but it is suggested that the Institute undertake for the present to standardize only those symbols which would be most general and widely used. The general principles which should govern selection are discussed, and there is given a list of suggested symbols covering some of the most important items. These are offered for criticism and suggestions in order to assist toward definite standardization.
\end{abstract}

$W^{1}$ HEN primitive man succeeded in transferring thought between two individuals beyond sight and hearing of each other, one of the first means was by symbols, or crude and roughly cut representations of objects. The limitations of the method need not be dwelt upon. Today, by the manipulation of twenty-six letters and ten figures into countless combinations, we can express upon the printed page anything which language may convey, but still we fined the method of our ancestors most convenient in many instances. Particularly in the diagrammatic sketches which are at once a convenience and a necessity to the electrical engineer, do we need to use and understand simple, easily made, and readily recognized symbols for the machines, instruments, and devices whose connections and uses we portray.

In these days, as never before in the world's history, it is unnecessary to argue the desirability of uniformity; of national and international standardization of all things connected with industry. To state the proposition is to have it accepted. Symbols constitute a language, and the more that language can be simplified and the broader its field of recognition, the greater will be its usefulness. We have the language, but there are many dialects; it may almost be said that there are many languages; and if the occurrence at Babel can be reversed, something will be added to the effectiveness of human endeavor.

A sub-committee of the Standards Committee, composed of Dr. P. G. Agnew, Mr. W. A. Merrill, and the writer, assisted by the effective co-operation of many particularly interested and informed engineers, have been investigating the possibility of bringing some degree of order out of this confused situation. It was first attempted to collect and tabulate the symbols used in this and other countries, with the idea that perhaps this alone would solve the problem by disclosing a weight of preference which could be accepted. The result was both instructive and confusing. It was found that there were plenty of symbols; almost every conceivable thing has been symbolized by some one, and most things by many; but of agreement there was very little.

Symbols now in use have been evolved as need arose. In most cases they have attempted to represent the

To be presented at the 8th Midwinter Convention of the A. I. E. E., New York, February 18, 1920. thing indicated closely enough to enable identification. This accounts for much of the confusion, as devices of the same class are so varying in appearance. It also accounts for much of the complication, the attempt to portray the object frequently resulting in something more like a picture or a working drawing than a true symbol. Slight variations in type or appearance are indicated by corresponding changes in the symbols, and in many instances the results are not, and never will be, interpreted except by the few initiated. In justice it should be said that some are so widely used as to be generally recognized, and these afford a foundation upon which to build. It should also be said that there has been found no reactionary desire to cling to the particular symbols now used, but on the contrary there is a generally expressed willingness to accept any thing upon which there can be general agreement.

There is practically no limit to the things which can be symbolized, and one of the first questions is to define the Institute's scope. Obviously the adoption of too many symbols would approach the situation of Chinese writing; only the specially trained would be able to interpret, and the aim would be defeated. There is also grave question whether the Institute is warranted, for the present at least, in going into special fields, or dealing with anything except of general application. It is believed that the Institute might properly standardize such symbols as will be used in papers which are presented to it, or in connection with diagrams of a general nature; but should not, unless by agreement with the interests affected, try to fix all symbols needed for special fields, such as full connection diagrams for switchboards, control equipments, or telephone, telegraph and radio apparatus. The National Electrical Contractors' Association, for instance, has a set of standard symbols, extremely useful to them, and regarding which they are unquestionably the best judges. It is to be hoped, of course, that the various special fields will use the standard general symbols and co-ordinate their special symbols with them.

It is suggested that the symbols included in a stands ard list should conform to the following principleso far as possible:

a. Be adapted to universal use.

b. Be simple and easily made. 
c. Be flexible; i.e., permit indication of as many things as possible without additional symbols and permit simple modifications or extensions for special cases.

d. Be indicative in appearance.

e. Be those most used at present.

In order to elicit suggestions and criticisms the list of symbols shown in Plates 1,2 and 3 are presented as conforming to the foregoing principles and covering some of the most important items.

1. Conductors. It is rather common to show conductors crossing, but not connected, as in Fig. 1, but

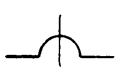

this makes the construction more

- difficult and seems unnecessary. The

Fic. 1 absence of a dot should be suffielectrical connection.

2. Earth Connections. This is quite generally used and seems to be satisfactory.

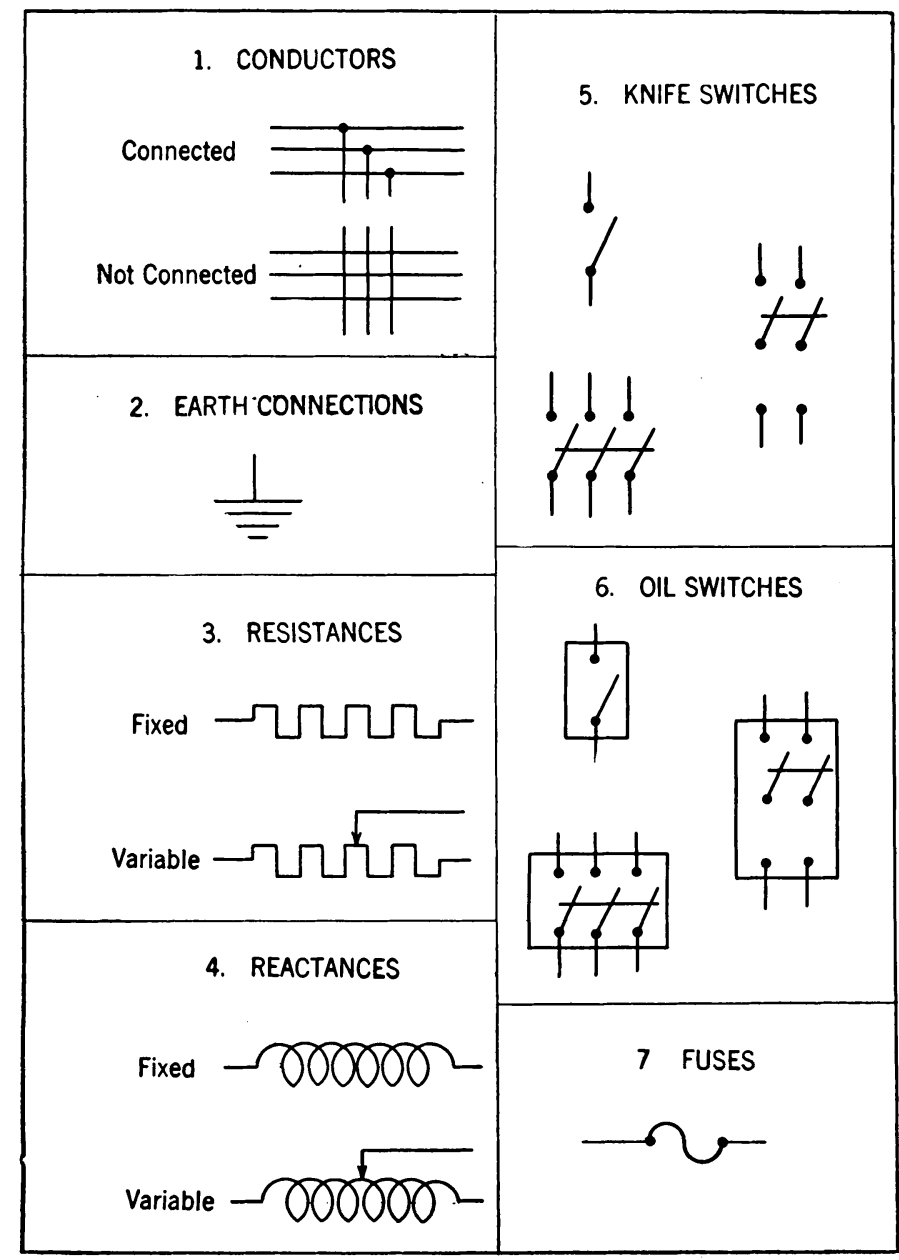

Plate 1

3. Resistances. 4. Reactances. 9. Windings. These three should be considered together because of the way they are confused in current practise, although it is important that they should have distinctive symbols. At present the zigzag line (9) is used rather indiscriminately to rer resent all three. The rect- angular line (3) is frequently used for resistance (sometimes for iron grid resistance only), but never for anything else, and seems to meet all requirements for a resistance symbol.

The succession of loops (4) is indicative of reactance and is widely used for that purpose in text books and elsewhere. Apparently the only objection raised against it is its alleged difficulty of construction. Its construction can be made difficult, but need not be. The loops in Plate 1 are drawn with one sweep of a compass, $5 / 6$ of a circle being drawn about each center except the end ones. No spacing is necessary; each new center is placed by eye just far enough outside the preceding arc to allow for the ink line. Drawn in this manner, which was independently developed by Mr. J. P. Buckley but apparently by others also, the looped figure is easier to construct than either of the others.

This leaves the zigzag line to denote windings of all kinds, thus the three as shown are submitted as a solution of perhaps the most perplexing of the problems involved.

5. Knife Switches. 6. Oil Switches. The convention for knife switches will probably be generally accepted, but the indication of an oil switch by putting a rectangle around the corresponding knife-switch symbol is probably novel. The advantages of the system are fairly obvious.

The English indicate live and dead sides of a switch by having the conductor terminate in an open circle at the dead side and in a full dot at the live side, but this makes construction difficult, may sometimes be misleading, and seems to be an unnecessary complication.

7. Fuses. This is the old symbol, designed, apparently, to represent a wire of soft metal twisted around two binding posts. Cartridge fuses are commonly shown as in Fig. 2 but this is not sufficiently general. The symbol shown on Plate 1 , constructed with two half circles, is easy
to make and quite distinctive.

8. Circuit Breakers. Existing symbols are mostly sketches of some particular type of breaker. The symbol suggested will probably be generally recognized, is sometimes used now, and is distinctive.

10. Transformers. The symbol for transformers will naturally follow if (9) (for windings) is accepted. Of course there ought to be further elaboration to permit indication of polyphase transformers and different bank connections, but that has not been undertaken as yet.

11. Rotating Machines. A circle, with conductors coming to the periphery, is used as the general symbol. Small rectangles, representing brushes, will indicate direct-current machines. Letters inside the circle will give any additional information desired. Mechanical connection is shown by a line joining the centers.

12. Meters and Instruments. This is the same as 
for rotating machines except the conductors will come to dots inside the circles.

13. Shunts. This is the symbol generally used. Sometimes the shunt circuit con-

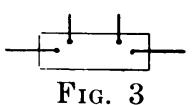
ductors are brought to a separate set of dots as in Fig. 3, but this seems to be an unnecessary complication.

14. Relays. A confusing multitude of symbols is now in use. The system suggested is uniform with that for machines and meters except that a rectangle is used.

15. Batteries. The general symbol is that commonly employed but it seems desirable to differentiate between primary and secondary batteries, and the suggestion is to put a rectangle around the "plates" to indicate a storage battery.

In using this symbol it ought to be understood that the long, thin line is the positive terminal, and this convention should be rigidly adhered to whenever polarity is of importance.

16. Lamps. These are almost universal.

17. Condensers. This is the most commonly used symbol and seems to meet all requirements.

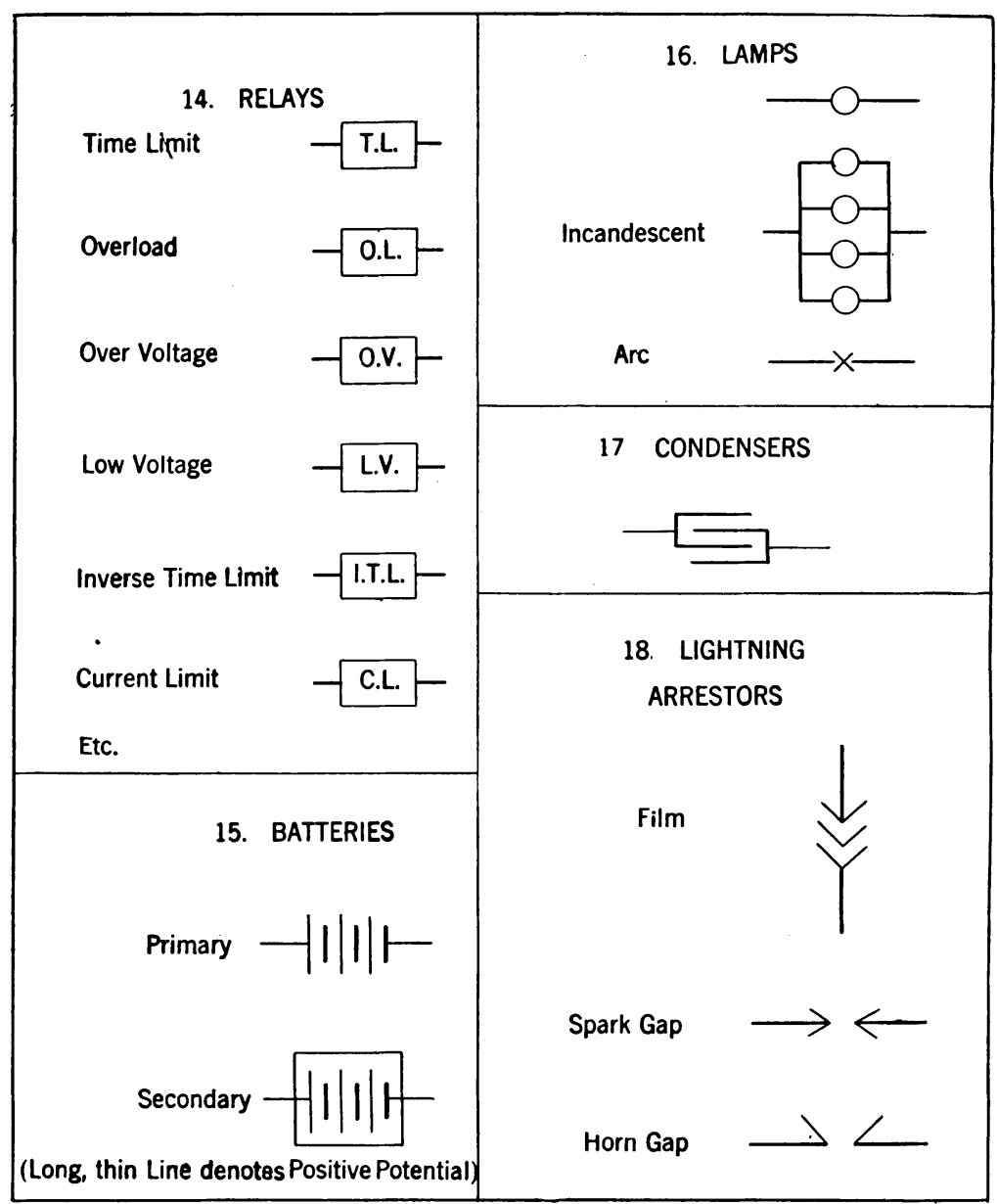

Plate III.

18. Lightning Arresters. There are many types and many symbols. The three symbols shown will doubtless be readily recognized and cover the most general cases.

\section{CoNCLUSION}

After all interests and all views have been fully considered, it is suggested that this group of symbols, modified as may appear advisable, should be included in the standards of the Institute, there to be added to from time to time as occasion warrants. It will, of course, be desirable to secure as much international agreement as possible. In this connection it should be said that the practise of other countries has been given full weight in determining the symbols herein suggested.

The author is indebted to his fellow subcommittee members and to many others for suggestions, and it is hoped that this paper will arouse general interest and bring forth criticism and constructive suggestions which will enable definite decisions to be reached at an early date. 